\title{
LE MODE DE SIGNIFICATION "IMMÉDIAT" OU PHYSIONOMIQUE
}

\author{
FELIX THÜRLEMANN \\ Université de Zurich
}

\section{Le phénomène et sa dénomination}

Dans l'essai théorique "Ueber die Formfrage" (sur la question de la forme) publie en 1912 dans l'almanach Der Blaue Reiter, le peintre Wassily Kandinsky convie le lecteur à un exercice mental':

Si le lecteur considere avec des yeux neufs n'importe quelle lettre de ces lignes, autrement dit s'il ne la regarde pas comme un signe connu faisant partic d'un mot, mais comme une chose, il ne verra plus dans cette lettre une forme abstraite créce par l'homme en vue d'une certaine fin - la désignation d'un son détermine -, mais une forme concrète produisant par elle-même une certaine impression extérieure et intérieure, indépendante de sa forme abstraite. En ce sens, la lettre se compose:

1. d'une forme principale - son aspect global - apparaissant (très grossièrement dit) comme "gaie", "triste", "dynamique", "languissante", "provocante", "orgucilleuse", ctc.;

2. de différentes lignes orientécs de diverses manières, produisant à leur tour une impression "gaic", "triste", etc.

Si le lecteur prend conscience de ces deux éléments, il éprouve aussitôt le sentiment que produit cette lettre en tant qu'être ayant une vic intérieure.

Dans ce passage, Kandinsky dispense avec une grande habileté didactique une leçon sémiotique élémentaire: un même donné sensible peut servir, suivant le "regard" qu'on y applique, de signifiant a deux signifiés complètement différents: le caractère d'imprimeric peut être considéré comme élément d'un système de représentation graphique ("désignation d'un son détermine") ou "comme une chose", un "être" qui, d'après Kandinsky, serait doté, à lui scul, d'"une vic intéricure"2.

La lecture des lettres "avec des yeux neufs" proposée par Kandinsky relève à notre avis d'un mode de signification fondamental qui règle dans 
une large mesure le rapport de l'homme aux objets tant artificiels que naturels du monde. Malgré tout, le phénomène sémiotique en question n'a pas encore trouvé de traitement théorique adéquat, et il manque un terme stable pour le désigner. Les psychologues parlent du mode de perception "physionomique" des choses, qui serait propre avant tout aux enfants et aux sauvages, mais aussi aux artistes ${ }^{3}$; les théoriciens des arts plastiques et de la littérature utilisent des expressions comme signification "immédiate" ou "directe"4. Roland Jakobson et Linda Waugh (1980:280), par exemple, attribuent aux sons du langage "la puissance de signifier immédiatement":

Cet enchantement du "pur son des mots", qui éclate dans les emplois expressifs, magiques et mythopoïétiques du langage, et par-dessus tout en poésie, non content de compléter et de contrebalancer le procédé spécifiquement linguistique de "double articulation", dépasse la dualité en conférant aux traits distinctifs cux-mêmes la puissance de signifier immédiatement. La voie médiate du sens va mème jusqu à disparaitre totalement dans les expériences poétıques du début de ce stècle, parallèles aux tendances abstraites en peinture et proches de l'élément magique de la tradition orale.'

Si l'école américaine de sémiotique n'a pas pu fournir une théorie du mode de signification en question, ceci s'explique sans doute par le fait que le modèle de Peirce est essentiellement une typologie des signes; la lecture physionomique des objets par contre repose comme tous les procès de signification élémentaires - Jakobson et Waugh l'ont indiqué - sur des unités plus petites que les signes manifestés. Les raisons pour lesquelles l'écule européenne de sémiotique de son côté n'a pas encore théorisé le phénomène ne nous paraissent pas d'ordre épistémologique, mais semblent résider dans l'origine logocentrique du modèle. Nous verrons cependant que déjà Hjelmslev a - quasiment à son insu - développé des éléments particulièrement féconds pour une théorie du mode de signification physionomique.

Dans ces quelques pages, nous nous fixons comme but (1) de décrire du point de vue de la sémiotique générale quelques éléments formels qui caractérisent le mode de signification "immédiat" ou physionomique et (2) de donner des indications stratégiques pour la constitution d'unc théoric adéquate du phénomèné ${ }^{6}$. Les exemples d'illustration seront surtout tirés du domaine visuel. Il faut cependant être conscient que toutes les substances de tous les sens peuvent être perçues selon le mode de signification pliysionomique. Celui-ci paraît jouer un rôle également important dans les arts visuels, dans les arts auditifs, la musique et la poésic, dans les "arts" culinaires, dans l'appréciation des vins et des parfums... 


\section{Eléments d'une caractérisation formelle}

Kandinsky n'a pas seulement esquissé une théorie de la signification physionomique, il en a également appliqué le principe dans l'interprétation de ses propres ouvres. Un passage, tiré de l'essai auto-interprétatif dédié à Composition VI de 1913, nous aidera à micux saisir la structure formelle du mode de signification physionomique. Le peintre y décrit les deux "centres" de son tableau (Kandinsky 1974:137)?:

Dans ce tableau, on voit deux centres:

1. à gauche, le centre délicat, rose et légèrement estompé, avec au milieu des lignes faibles et incertaines;

2. a droite (un peu plus haut que celui de gauche) le deuxidme, plus grossier, rouge-bleu, légèrement déton[n]ant, avec des lignes acérées quelque peu méchantes, fortes et très précises.

D'après Hjelmslev (1971), il y a deux conditions essentielles qui doivent être remplies pour qu'on ait affaire à un langage, c'est-à-dire à un système sémiotique: (1) il faut pouvoir procéder à une description indépendante de la forme de l'expression et de la forme du contenu, (2) il faut pouvoir déterminer la relation entre les deux formes.

Si on examine les termes employés par Kandinsky dans la description de son tableau, on se rend compte qu'ils peuvent être regroupés en deux classes, des termes descriptifs de nature objective (comme "estompé", "rouge-bleu", "précises") et des termes qui possèdent un caractère évaluatif accusé (comme "délicat", "grossier", "méchantes"). Notre proposition tend à considérer les termes objectifs comme une description du plan de l'expression, les termes évaluatifs comme une description du plan du contenu. Pour la dimension chromatique, qui seule possède une caractérisation suffisante par des termes neutres, on peut ainsi établir le schéma suivant:

\begin{tabular}{|c|c|c|}
\hline & expression & contenu \\
\hline $\begin{array}{l}\text { centre } \\
\text { à gauche } \\
\text { centre } \\
\text { à droite }\end{array}$ & $\begin{array}{l}\text { teinte non saturéc (rose), } \\
\text { limites effacécs (estompé) } \\
\text { teintes saturées à articulation } \\
\text { discrd̀te (rouge-bleu) }\end{array}$ & $\begin{array}{l}\text { délicat } \\
\text { grossier, légère } \\
\text { ment détonnant }\end{array}$ \\
\hline
\end{tabular}


La description de Kandinsky permet d'observer un certain nombre de traits formels:

(1) Le mode de signification physionomique obéit au critère de nonconformité entre forme de l'expression et forme du contenu qui selon Hjelmslev caractérise les langages proprement dits. Dans la description étudiée, le contenu "délicat" est attribué à un ensemble de traits plastiques (/teinte non saturée/ + /limites effacées/). Le centre à gauche manifeste un "vocable" visuel du mode de signification physionomique qui semble posséder une validité générale à l'intérieur de la culture occidentale. (Il suffit de penser aux photographies et aux films de David Hamilton, qui l'utilise jusqu'à l'excès).

Dans son étude expérimentale sur la perception esthétique des couleurs, J.G. von Allesch a pu démontrer que même les teintes chromatiques apparemment "simples" sont toujours conçues comme des paquets de traits (relevant de catégories telles que la chromaticité, la saturation, la luminosité).

(2) La lecture de Kandinsky s'applique à un syntagme (à deux "centres" en position topologique comparable). La structure syntagmatique de l'objet perçu possède sans doute une fonction essentielle pour la détermination des catégories pertinentes du plan de l'expression. (Dans l'exemple étudié, le rose s'oppose au rouge-bleu comme une teinte non saturée à des teintes saturées).

Les deux principes mentionnés - décomposition des unités de la manifestation en traits contrastifs, détermination des catégories pertinentes à partir de la structure syntagmatique englobante - sont aussi valables pour l'analyse du phénomène du "symbolisme vocal", qui peut être considéré comme un cas typique de signification physionomique dans le domaine auditif. Si les études phonostylistiques traditionelles $n$ 'ont pas abouti à des résultats convaincants, c'est qu'elles reposent - contrairement aux travaux exemplaires de Jean-Claude Coquet - sur les sons du langage comme unités d'analyse 8 .

(3) Dans son étude "Sur la question de la forme", que nous avons déja citée, Kandinsky postule pour la signification physionomique une structure hiérarchique; l'effet de sens global d'un tableau ne peut pas être conçu comme la somme des effets de sens physionomiques de ses constituants (Kandinsky 1974:156) 9 :

Les lignes prises isolément peuvent ètre "gaies", tandis que l'impression globale (elément 1) peut produire un effet de "tristesse", etc. Les différents mouvements du second élément sont des parties organiques du premier. 
Dans toute mélodie, sonate ou symphonie, nous observons la même subordination des éléments isolés à un seul effet d'ensemble. Et nous pouvons dire autant d'un dessin, d'une esquisse, d'un tableau.

3. Les deux principaux modes de description: synesthésique et caractérologique

Ce qui singularise le mode de signification physionomique est le fait que toute description des effets de sens qui se veut quelque peu précise doit faire appel à un vocabulaire indirect. Les interprètes emploient, outre une terminologie caractérologique - celle qui apparaît dans les textes cités de Kandinsky ("gai", "triste", "dynamique", etc) —, une terminologie synesthésique. Un même parfum par exemple peut indifféremment être décrit comme "envoûtant" ou "doux", une même couleur comme "aggressive" ou "chaude"10.

Louis Hjelmslev a soulevé le problème du discours descriptif indirect dans son article "La stratification du langage" de 1954. Hjelmslev y développe la thèse que toute substance peut être décrite selon plusieurs "niveaux", qu'il appelle respectivement niveau physique, niveau socio-biologique et niveau d'appréciations collectives." Le linguiste danois n'envisage nulle part, comme nous le faisons, la possibilité de considérer le "niveau d'appréciations collectives" comme le plan de contenu d'un mode de signification autonome. Cependant, les problèmes de description mentionnés par Hjelmslev se posent également pour le mode de signification physionomique (1954:63s):

Il paraît évident que, puisqu'il s'agit de degager des appréciations relativement naïves, et dont le seul fondement "théorique" est à chercher dans ce que nous avons appelé le "corps de doctrine" adopté dans les opinions de la socićte, la "métn-langue" dont une telle discipline pourrait se servir pour atteindre ses buts ne saurait être que le langage de tous les jours. [...] risquons au hasard quelques exemples relativement problables tels que. pour le français, 'clair': 'sombre', 'fort': 'faible', 'long': 'bref', 'haut': 'bas', 'pesant': 'leger', etc. Quoi qu'il en soit, une telle etude demande comme préparatif indispensable un examen du système d'adjectifs de la langue considerée et [de la] substance de contenu qu'ils comportent. Elle demanderait en outre tout un nombre d'autres preparatifs qui, à l'état actuel des recherches, ne sont point, il faut l'avouer, a la portée immédiate du chercheur. II faut y compter bien des faits d'ordre psychologique, y compris, entre autres choses, ceux de la synesthésie.

Nous voudrions ajouter à ect inventaire de problèmes à traiter l'étude du 
système caractérologique propre à la langue et à la culture de l'interprète. Une analyse parallèle des systèmes de description synesthésique et caractérologique pourrait, à notre avis, permettre de remplacer le vocabulaire "naïf" dont on se sert d'ordinaire pour décrire les effets de sens physionomiques par un vocabulaire construit. Ce n'est qu'avec une terminologie réduite qu'il sera possible de décrire d'une manière contrôlable les relations entre formes de l'expression et formes du contenu propres au mode de signification physionomique 12 .

\section{Propositions pour une stratégie de recherche}

Nous espérons avoir pu montrer que le mode de lecture physionomique des objets du monde peut être considéré comme un véritable langage dans le sens de Hjelmslev, dont l'analyse systématique devrait dorénavant faire partie constitutive du projet sémiotique. Dans la plupart des textes artistiques traditionnels, le mode de signification physionomique n'est pas le scul en jeu: dans la poésie, il se manifeste parallèlement à la dimension de signification sémantique, dans les arts plastiques, il s'ajoute à la signification thématico-figurative de nature iconographique. $\mathrm{Si}$, comme nous l'admettons, le langage physionomique possède un statut sémiotique autonome, son contenu peut, dans les textes particuliers, entretenir tous les types de rapports logiques avec le contenu sémantique ou iconographique: du parallélisme de renforcement jusqu'à la contradiction ironique. Il est donc nécessaire de distinguer clairement, dès le début de l'analyse, les différentes dimensions de signification ${ }^{13}$.

Les problèmes d'analyse que pose le mode de signification physionomique semblent être les mêmes que ceux rencontrés lors de l'étude des phénomènes regroupés sous le terme de connotation. Dans ces pages, nous avons évité à dessein ce concept, qui non seulement d'après l'étymologie, mais aussi dans la théorie hjelmslevienne implique l'idée de subordination hiérarchique. L'exemple de la peinture abstraite de Kandinsky montre que la signification physionomique peut, dans une esthétique donnée, jouer un rôle primordial. Dans la phase actuelle des recherches sur le langage physionomique, il nous semble profitable de se concentrer sur l'étude de phénomènes où celui-ci joue un rôle dominant, sinon exclusif, tels la peinture abstraite, la musique ou le "langage" des parfums par exemple.

Des considérations stratégiques invitent également à privilégier pour le moment l'analyse de textes. Les cas concrets, comme la description de Kan- 
dinsky que nous avons étudiéc, montrent que la structure syntagmatique des textes joue un rôle décisif dans le procès de constitution des catégories de l'expression pertinentes pour la lecture physionomique. Celle-ci est probablement beaucoup moins sujette à l'arbitraire qu'on ne le soupçonne généralement.

Mais d'autre part, il est nécessaire d'élever l'étude du mode de signification physionomique à un niveau général qui dépasse les domaines d'étude traditionnels. Un des grands champs de la future recherche sémiotique, celui des langages syncrétiques, demande la constitution d'une théorie commune ${ }^{14}$. Même si les problèmes d'articulation de la substance de l'expression se posent différemment dans les divers domaines sensoriels, le sens physionomique est un, comme le prouve l'existence d'un même langage descriptif caractérologique et synesthésique qui nous permet de parler de la signification que suscite "immédiatement" l'ensemble des objets du monde sensible.

\section{NOTES}

1) Nous citons le texte d'apris la traduction de Jean-Paul Bouillon (Kandinsky 1974:155). Pour les éditions du texte original allemand voir Bouillon in Kandinsky 1974:296ss

2) Greimas (1980:254) se sert, lui aussi, des lettres de l'alphabet pour Illustrer ce même phénomène. Gretmas oppose la lettre comme élément d'un système d'écriture al la lettre comme élément d'un système de représentation logeque.

3) Cf. Werner (1953:45,47): "Indem so die Dinge tellhaben an dem dynamischen Vollzug. durch den sie gefasst werden, an dem affektiven und motorischen Gebaren des Subjekts. erschenen sie dem naiven Menschen vielfach ganzlich anders als uns. Dee Dinge sind nicht sachloch,

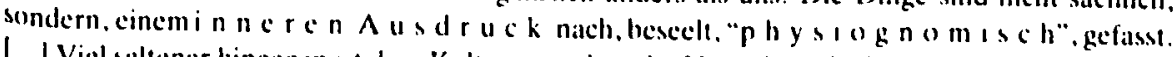
I. | Viel seltener hingegen ist dem Kulturmensehen die Umwelt nach cinem inneren Ausdrucke, durch ihren physiggnomischen (harahler, gegehen Besspelswese ist des dort der Fall, wo er eine Landschaft in asthetischer Wirkung fasst In solchen Fillen tritt cine ganz andere Art des Erlebens auf. Man sucht und findet gewisse rmassen (iesichter in der Landschaft; man erlebt se Physiognomisch: eine landschaft ist heiter oder traurg. spoutisch oder grausig, erhaben. heilig oder holliseh usw. Nur besondere Gegenstinde der Erkenntnis sund dem heutigen Kulturmenschen allgemein physiognomisch gegelen' die Afenschen selbse in diren (iesschtern und Korpern 1... Man findet gluck licherweise physwognomisches I: rletien nodh bes l:rwachenen vor, die jenen allgemeinen Typus reprasentieren, den ich den "Ausdrucksmensehen" nemnen möchte; der wahre Künstler erweist desen Typ in besonderer Reinheit und zwingt durch sein Werk den Betrachter.

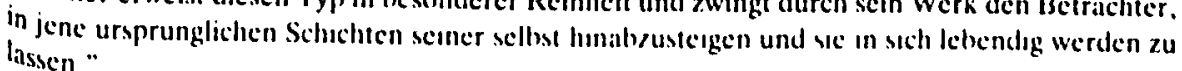

4) Cf. Gauguin (1946:45): "Tous nos cing sens arrivent directement au cerveau impressionnes par une infinité de choses ct qu'aucune éducation ne peut détruire. J'en conclus qu'il y a des multipes nobles, menteuses, etc. |... | les couleurs sont encore plus explicatives quoique moins multiples que les lignes par suite de leur puissance sur l'ocil. II y a des tons nobles, d'autres communs, des harmonies tranquilles, consolantes, d'autres qui vous excitent par leur hardiesse " 
5) Voir également Jakobson/Waugh (1980:270) “ “En poésie, les sons du langage manifestent spontanément et immédiatement leur propre fonction sémantique."

6) Une première tentative danalyse sémiotique du mode de signification physionomique a été fatte, sous lc nom de description ou lecture évaluatıve, in Thurlemann 1982:70, 82s, 90.

7) Le lecteur désireux de confronter le texte de Kandinsky avec l'objet décrit trouvera des reproductions en coulcurs de Composition VI dans Roethel 1977:pl.19; Kandinsky 1980: fig.44 et RoethelBenjamin 1982:469.

8) Pour une analyse phonostylistıque de deux couples de vers d'Apollinaire, voir Coquet (1973:125s). Voir également les consıdérations p. 100s et 107s eł un chapitre dans la contribution du même auteur au recueil Essaus de sémiotique poétique (Coquet 1972:32-34). La critique de l'analyse phonostylistique par Van Den Berghe concerne seulement des études qui prennent l'unité du son comme point de départ.

9) Pour une analyse de la seconde partic de l'auto-interprétation de Composition VI, où Kandınsky décrıt la constıtution successive de l'effet de sens global "objectivité" à partır des effets de sens de configurations contrastıves, voir Thurlemann 1985.

10) Pour le domaine des couleurs, l'étude expérımentale de von Allesch (1925) fournit un matérıel extrêmement riche de descrıptıons en langue allemande.

11) Greimas et Courtés (1979:189s), en évitant le terme de "niveau". distınguent trois "instances de substance" ou "mode de présence pour le sujet connasssant".

12) Lindekens (1971) a proposé une analyse extrêmement intéressante de différentes familles de caractères typographiques selon le mode de signification phystonomique. L'auteur cherche à déterminer pour chaque famille les traits de l'expressıon pertınents et de les corréler à une figure de contenu sous la forme d'une combinatoire d'un nombre réduit de traits caractérologiques. Les résultats de l'analyse se trouvent cependant appauvrs à la sutte de l'emploi d'une méthodologie trop quantitative, le "différentiel sémantique" de Ch. Osgood.

13) Bon nombre de lectures phonostylistiques critiquées par Van Den Berghe (1976) ne font que projeter le sens sémantique des mots ou des vers sur leur forme phonétique. Seuls les lecteurs particulièrement sensibles, tel un Mallarmé, ont été capables de ressentir des contradictions entre le sens physionomique et le sens sémantique des unités linguistıques. Voir le commentaire sur "jour" et "nuit" dans "Crise de vers" (Mallarmé 1945 369) et l'étude de Genette (1968).

14) Pour une première présentation de la problématıque, voir Foch (1983).

\section{RÉFÉRENCES}

Allesch, G.J. von. 1925. 'Die ästhetische Erscheinungsweise der Farben', Psychologische Forschung 6: 1-91, 215-281.

Coquet, Jean-Claude. 1972. 'Poétique et linguistique', in A.J.Greimas (éd.), Essais de sémiotique poétique, Larousse: Paris: 26-44.

-..-.. 1973. Sémiotique littéraire: contribution à l'analyse sémantique du discours, Jean-Pierre Delarge/Mame: Paris.

Floch, Jean-Maric. 1983. (éd.) Sémiotiques syncrétiques, Actes sémiotiques: 
Bulletin VI.27.

Gauguin, Paul. 1946. Lettres de Gauguin à sa femme et à ses amis, éd.

M.Malingue, Bernard Grasset: Paris.

Genette, Gérard. 1968. 'Le jour, la nuit', Langages 12:28-42.

Greimas, Algirdas Julien. 1980. 'Semiotica figurativa e semiotica plastica', Filmcritica 307-309:253-267.

Greimas, Algirdas Julien ct Courtés, Joseph. 1979. Sémiotique: dictionnaire raisonné de la théorie du langage, Hachette: Paris.

Hjelmslev, Louis. 1954. 'La stratification du langage', Word 10:163-188, cité d'après L. Hjelmslev, Essais linguistiques, Ed. de Minuit: Paris 1971:44-76. -... 1971. Prolégomènes à une théorie du langage, Ed. de Minuit: Paris. Jakobson, Roman et Waugh, Linda. 1980. La charpente phonique du langage, Ed. de Minuit: Paris.

Kandinsky, Wassily. 1974. Regards sur le passé et autres textes 1912-1922, éd. établic et présentée par Jean-Paul Bouillon, Hermann: Paris.

…. 1980. Die Gesammelten Schriften, vol.1, éd. H.K. Roethel et J.HahlKoch, Benteli: Berne.

Lindekens, René. 1971. Sémiotique de l'image: combinatoire des traits pertinents dans une image minimale, au niveau de cinq familles de caractères typographiques, Documents de travail - Université d'Urbino.

Mallarmé, Stéphane. 1945. 'Crise de vers', in: Oeuvres Complètes, Gallimard: Paris.

Rocthel, Hans K. 1977. Kandinsky, Nouvelles Editions Françaises: Paris.

Roethel, Hans K. et Benjamin, Jean. 1982. Catalogue raisonné des peintures à l'huile, vol. 1:1900-1915, Flammarion: Paris.

Thürlemann, Félix. 1982. Paul Klee: analyse sćmiotique de trois peintures, L'Age d'Homme: Lausanne.

…. 1985. 'Analisi di una autointerpretazione di Kandinsky: il modo di significazione sinestetico-fisiognomico', Archivio storico del Ticino.

$V_{a n}$ Den Berghe, Christian Louis. 1976. La phonostylistique du français,

Mouton: La Haye/Paris.
Werner, Heinz. 1953. Einführung in die Entwicklungspsychologie, $3^{\mathrm{e}} \mathrm{ed}$., J.A.Barth: Munich. 